\title{
Differential Effects of Analogy Types on Retrievability and Inferential Induction in Text Comprehension
}

\author{
Stephen Ntim, PhD; M.Phil; B.Ed; M. A \\ Faculty of Education, Catholic University of Ghana \\ P.O. BOX 363, Fiapre-Sunyani, B/A Ghana, W/Africa \\ Email:stephenntim58@yahoo.com
}

Doi:10.5901/ajis/2013.v2n1p109

Abstract

In the experiments presented in this study, the researcher examined the possibility and found evidence suggesting the different uses of analogy in structuring, reminding and understanding novel information. Specifically, when given series of written passages that either shared structural similarity, literal similarity, surface attributes or first order relations, individuals were likely to make interpretations that paralleled structural information from a previously read analogous scenario. In contrast with the great majority of existing research, as well as with some common conceptions about analogy use, this interpreting was done in the absence of direct didactical intervention other than text comprehension. Strikingly participants were able to draw inferences. Findings from the experiments seem to suggest that surface attribute and literal similarity were quite influential in remindings and access whereas structural similarity appeared to be more sensitive to inference-drawing. The data were taking as supporting the evidence that a) different types of similarity affect analogies differently; b) that inference-drawing and elaboration can take place automatically in text comprehension.

Key Words: Analogy; surface similarity; literal similarity; recall; inference, text comprehension

\section{Introduction}

Reasoning by analogy is a fundamental component in human cognition. Analogy provides a tool for thought and explanation. Its role has been critical in scientific discoveries and creative thinking. It is precisely because of this that its fundamental role in reasoning has been the focus of attention in cognitive psychology. It is through analogy that many classic scientific discoveries were made. A well known example is that of Archimedes in the 3rd century B.C. When asked to determine whether a base metal had been substituted for gold in an intricately designed crown, although the weight per volume of pure gold was known, the crown was so ornate that its volume was impossible to measure. He was unable to solve this solution until he went home and stepped into his bath: he saw an analogy between the volume of water displaced by his body as he got into his bath, and the volume of water that would be displaced by the crown. The problem was solved.

Another classic example of analogy in science is Kepler's analogy between religion and the relationship between the motion of the planets and their distance from the sun. It was concerned primarily with three problems, namely, the number, size and motion of the planets and the analogy between the stationary objects, namely, the sun, the fixed stars, and the space between them, with God the Father, the Son and the Holy Ghost. The analogy mapped out as follows: 'The sun in the middle of the moving stars, himself at rest and yet the source of motion carries the image of God the Father and the Creator. He distributes his motive force through a medium which contains moving bodies, even as 
the Father creates through the Holy Ghost. Thus analogy eventually led Keppler to an operational theory of celestial mechanics that resulted in the notion of gravity.

Thus analogies are important in learning, problem solving and discovery. The paradox however is that developmental psychology, for example, has not given central role to analogy. Goswani (1992) has offered the explanation that this neglect was partly historical: namely, the over-emphasis on Piagetian theory which asserts that reasoning by analogy was a late-developing skill around II-I2 years of age. This traditional view was supported by research in psychometrics. Psychometrics research had shown that many children, even at II-I2 years of age, were unable to complete simple analogies like pig: boar: dog:? (wolf). When given these analogies, younger children typically produced responses, like pig:boar : $\operatorname{dog}$ : cat (Goswani,I992, p. 4).

Much of human experience however is essentially analogical. Analogy entails mapping the problem representation (target) into a structurally similar schema (base), which has been learnt through experience. Two sources of difficulty are foreseen in analogical reasoning: mapping problems to inappropriate schemas, and processing loads imposed by mapping; the more complex the mapping, the greater the load. Gentner, (1983) for example, defines analogies as mapping from a base to a target. In the simple proportional analogy, "human is to baby" as "horse is to foal" is the target. Elements in the base are mapped into the target. In this case, all mappings are bi-directional, unless indicated otherwise, so it does not matter whether they are described from base to target or the reverse.

In the thinking of Halford (I992) when "human" is mapped into "horse" and "baby" into "foal", the relation of "parent of" in the base there corresponds to the same relation in the target. In other words, the corresponding relation in the base and the target need not be identical (Halford, 1992, p. 194). For example, not all attributes of base and target elements are mapped such as the human attribute "walks on two legs" is not mapped to horse. The implication here is that relations are mapped selectively. It is these relations that enter into coherent structure that are more likely to be mapped. In the thinking of Gentner (1983) this is referred to as the principle of systematicity. Thus according to this view, the important thing in analogy is to establish that the base and the target are in structural correspondence.

Thus, the one most important issue that underscores all the research work on the different models of analogy, whether, it is from the point of view of pragmatic reasoning (Holyoak), componential reasoning (Sternberg) or the structural model (Gentner) is the important role of similarity. Analogical inference (and transfer for that matter) can take place to the effect that the subject can see salient features or similarity between the base or one's knowledge and the target or the transferred task.

\section{Statement of Problem}

Research findings corroborate the hypothesis that there is a correlation between analogy types and retrievability and inference-making. Ross (1989) in a study of transfer in problem solving measured not only the proportion of correct solution, but also the proportion of remindings, as measured by whether subject wrote out a prior formula. This allows a contrast to be made between solution rate (a measure which presumably includes mapping, adaptation, evaluation and drawing of inferences and reminding rate) which was relatively strongly affected by surface similarity. Novick (1988) gave both novice and expert mathematicians' problems to solve that included both surface similarity distractors and remote analogies, followed by later target problem. Initially both novices and experts retrieved surface similarities, but experts were quicker to reject initially incorrect retrievals, suggesting stronger effects of domain knowledge in mapping and evaluation than in retrieval. Gick and Holyoak (1983, 1987) demonstrated that a literally similar prior story was retrieved more often than on mapping and use. It is 
within this context of subjects attending more to surface similarity rather than structural that has made other scholars in cognitive psychology and education deny that transfer is spontaneous.

"Transfer is rare, and the likelihood of its occurrence is related to the similarity between two situations" (Detterman \& Sternberg, 1993, p. I5).

and

"There is no good evidence that people produce significant amounts of transfer or that they can be taught to do so. There is on the other hand, substantial evidence and an emerging Zeitgeist that favours the idea that what people learn are specific examples... I subscribe to the principle that you should teach people exactly what you want them to learn in a situation as close as possible to the one in which the learning will be applied. I don't count on transfer and I don't try to promote it" (Detterman \& Stemberg, I993, pp. I7).

Notwithstanding the above claim, other research as mentioned above do claim that there is not only transfer, but that there are indeed different types of analogies that exert differential effects on retrievability and inference-making (Gentner, Ratterman \& Forbus 1993). Thus, if these studies that make such claims are anything to go by, then one can reasonably imply that some types of analogies can enhance spontaneous transfer especially in the area of making inferences in text comprehension. As these and countless other examples demonstrate, analogy provides a useful tool for reasoning about poorly understood situations, solving difficult problems, and making plausible inferences about unknown properties, behaviors, and characteristics (Gentner \& Markman, 1997; Holyoak \& Thagard, 1995; Hummel \& Holyoak, 1997).

This research examines more closely the roles of similarity in transfer; specifically how the different types of analogies (surface similarity) and structural analogy (structural similarity) affects retrieval access and inference-making with reference to text comprehension. However, most research works on analogy with the exception of few have arrived at evidence with some form of instructional intervention and training from a previously read scenario in problem solving. In this study however, while assessing the differential effects of analogy types on retrievability and inferential induction in text comprehension, it also seeks to investigate whether or not there is spontaneous evidence of inferential transfer in text comprehension without didactic intervention. In short, whereas many researchers arrive at conclusion through an exposure of experimental groups to didactic intervention, this study seeks evidence non-intentionally (Day \& Gentner, 2007).

\section{Research Questions}

In the light of this defined problem, this research attempted to respond to three fundamental questions:

I)How is accuracy of recall of text dependent on the degree of surface similarities? (surface match)?;

2)Do people fail to infer structurally appropriate analogies in text comprehension?

3)Why is transfer likely to occur to the extent in which base and target share surface similarity?

\section{Hypothesis}

The fundamental hypothesis of this research is this:

a) Individuals are more likely to recall passages that shared surface similarity more than those with structural similarities; 
b) With respect to inference-making, individuals, who made use of structural similarities, are more likely to make better inferences.

c) Analogical transfer is likely to occur to the extent in which base and target share surface similarity?

Thus, the ability to transfer from a prior (base) knowledge to a target knowledge for fluent text comprehension is a function of two types of analogy each of which has differential impact in text comprehension: These two types of analogies are: a) remote analogy and b) within-analogy. Each has different cognitive function. Whereas remote analogy is crucial to inference-making and text elaboration, within-analogy aids access to retrieving surface contextual features. Each of the two types of analogy is differentially crucial for retrievability and inference.

\section{5- Method of testing the hypothesis}

This hypothesis was tested experimentally by exposing Second Year Education students in the Catholic University of Ghana to written passages in a text in which some of the passages were superficially very similar, while others were on the surface very dissimilar and yet shared structural similarity. Other facts in some of the passages were left unstated or ambiguous.

Subjects: 30 Second Year Education students in Psychology Class, Catholic University of Ghana.

\section{I Design and Materials}

Similarity types were varied within the subjects. In all, subjects read a total of 24 short stories in groups of three that is each group of 10 students read 8 stories. The first and the last stories were filler stories in addition to the main 6 stories in each group. Measurement was on the dimension of students recall along the following scales: ratings of their recall, proportion of recall rated above criterion, and proportion of recall of significant key word in each story. The stories were short ones of about two or three paragraphs. Each of the main I8 stories contained the following: an original story, three matching cue stories which differed in amount and level of similarity that they share with the original. All cues shared identical or nearly identical first order relations (e.g. events and actions) with the original story. They differed in other level of shared similarity.

There were three sets of cues: a) analogy cues, b) surface similarity match cues, and c) first order match cues. Analogy cues consisted of common higher order relational structure added to the first order relational matches; surface similarity match cues (SS) object matches were added to the first order relations matches. The higher order relational structure (which had to do with causal relations or plot structure differed; first order match (FOR) cues consisted only of the first order relation match. Each subject received only one matching cue story for each of the 18 main stories. All subject received the same memory set with differences only in the type of story used in the cue set. Comparability was ensured by making the cue stories as similar as possible. The use of identical words was avoided in order to ensure avoidance of lexical meanings in cueing.

Reminding task: subjects were tested in groups of three consisting of 10 students in each group in two separate sessions. In session one, students read a booklet containing the I8 original stories plus the 6 filler stories. All subjects read the same 24 stories in different semi-random orders with the order to read the stories carefully so that they would be able to remember them carefully. They took 35 minutes for this task. The second session took place later the same day without any didactic intervention. Students received booklet that contained the 18 stories they read earlier. Each workbook consisted of six surface similarity cues (SS), six analogy (AN) cues and six first order rules (FOR) cues. They were told that for each cue they were to write any original story for which they reminded. If they were reminded of 
more than one, they should write the one that best matched the current story: they were asked to include as many details as possible that they could remember.

Inference-drawing task: After completing the reminding task, students were asked to draw inference in the second session. The task was this: they were given the same stories as in the first session and were asked to rate six surface similarity (SM), six analogy(AN) and six first order rule (FOR) matches in the same order as in the reminding task. They were asked to rate each pair for the inference of the match explaining inference as the situation in which the essential aspect of the stories match for one to draw conclusions about the second story from the first.

Scoring: was done using 0-5 mean scale as follows: 5= All important elements of the original and many details; $4=$ all important elements of the original and some details; $3=$ all important elements of the original but very few or no details; $2=$ some important elements of the original; others missing or wrong; I= some elements from the original but not enough to be certain that the subjects genuinely recalled the original; $\mathrm{O}=$ no recall or different story.

In the third research question, subjects were the same as in questions $I$ and 2 with the same materials except that only AN matches and two kinds of surface matches were used. Each subject was given seven analogy matches (AN) and seven superficial matches. For half of the subjects the superficial matches shared only object descriptions and not first order event nor causal structure. The other half received SS matches of the same kind used in the previous experiments which shared first order relations as well as objects. Each subject in addition received six LS matches (the same six across all subjects. Procedure was also the same as in questions I and 2 except that this time round the keyword scoring was dropped. First session: subjects read twenty original stories and 12 filler stories. Second session: Later they were given 20 matching stories in the reminding task.

\section{Clarification of terms and acronyms}

As used in this study, the term literal similarity means that both relational predicates and object attributes share some similarities; whereas surface similarity is mere appearance matches in which only object-attributes and low order relations are shared. Analogy is used in this study to mean structural similarity as opposed to surface similarity or literal similarity. The same term is also used synonymously as 'across-domain analogy' or 'remote analogy' which appears to be sensitive 'inference-making' and elaboration in text comprehension in contradistinction to 'within-domain analogy' which is also used synonymously as 'surface similarity'. Thus within-domain analogy or surface similarity seems to be compatible with recall. The acronyms used in the experiments are: AN= Analogy; SS= Surface similarity; $\mathrm{LS}=$ Literal similarity; FOR $=$ First order rules

\section{Theoretical framework}

Similarity is universally acknowledged to be central in transfer. Research suggests that its role is complex. People solve problems better if they have solved prior similar problems. This applies to both children and adults. One of the most enduring findings in the field is that transfer promotes reminding. The most fundamental ideal required to understand transfer is that two tasks may differ and yet share some common components. This shared components provide the basis for inter task transfer. This notion of common components also sometimes referred to as 'the theory of identical elements' was first articulated by Thorndike in 1903. It is called 'salient features' or 'overlapping rules' in cognitive literature.

Theorists explain that the continuous notion of similarity can be reduced to a function of discreet components: two situations are similar in so far as they share many common components. To a first 
approximation, more common components will constitute greater similarity and therefore lead to greater transfer (Gick \& Holyoak, 1987, p. 16). To be able to predict the overall magnitude of transfer and its direction (positive or negative), a more refined analysis of similarity is required. These distinctive aspects of transfer can be related to the impact of different types of similarity on the retrieval verses application of previously acquired knowledge.

Specifically, what Gick and Holyoak are proposing here is that perceived similarity is a function of any salient similarity of two situations. This perceived similarity will in turn affect retrieval of the representation of the training situation during transfer task: the greater the perceived similarity of the two situations, the more likely it is that transfer will be attempted. If transfer in fact is attempted, the direction of the transfer will be determined by the similarity of the two situations with respect to features causally relevant to the goal or required response in the transfer task. It is crucial to understand the distinctions between the pragmatic approach to analogy in problem solving and that of the structural approach. The former is talking about 'features causally relevant to the goal' that will constrain the perceived similarity whereas the latter is emphasising the principle of systematicity.

Components of a situation that are causally or functionally related in outcomes or goal attainment is referred to by Holyoak et al. (1987) as structural and those not so related will be termed surface (Holyoak, 1985). These authors argue within the context of problem solving analogies that salient common components of either a surface or a structural nature will increase the likelihood that a problem solver will relate the two situations to each other. In other words, salient surface or structural components will affect perceived similarity. Conditional on transfer being attempted at all, shared structural components will tend to yield positive transfer, as the solution of the initial problem is transferred in an appropriate way to the transfer task.

The claim that analogy involves a mapping of information is a general assumption that is shared by most theories of analogy. The only difference however is that the factors that influence the mapping differ across theories. One of the most influential theories of how people use analogous solutions is the structure-mapping theory proposed by Gentner in 1983. The theory was primarily developed to account for mapping knowledge from a base domain onto a target domain that consisted of different objects, such as comparing an atom to a solar system. Because the objects differed, Gentner argued that it is the relations among the objects rather than the attributes of the objects that determined the mapping.

Thus, cognitive processing of analogies has been a fertile and productive area for research over the last two decades. There is substantial consensus on the fundamental processes involved (Gentner, 2003; Gentner, Holyoak, \& Kokinov, 200I; Gentner \& Markman, I997; Holyoak \& Thagard, I989; Hummel \& Holyoak, 1997; Kokinov \& French, 2003). A key characteristic of analogical theories is the emphasis on structured representations that specify the relations between elements. For example, in structuremapping theory (Forbus, Gentner, \& Law, 1995; Gentner, I983, 2003; Gentner \& Markman, 1997), the comparison processes act to achieve a maxima structurally consistent alignment between two representations. Structural consistency entails that the correspondences between the elements and relations in two representations must satisfy one-to-one correspondence (an element in one representation may be mapped to at most one element in another) and parallel connectivity (if two predicates correspond, their arguments must also correspond). Once a structural alignment has been established, candidate inferences are projected. These are additional elements that are connected to the common system in the base (or source) structure but are not yet present in the target (Clement $\&$ Gentner, I99I; Markman, 1997). Importantly, in structure-mapping, such inferences arise automatically via a structural pattern completion process (Day \& Gentner, 2007).

Gentner, Ratterman and Forbus (1993) in a similar study examined the roles of similarity and how different types of analogies or similarities affect retrieval access and what they call inferential soundness'. They attempted to isolate and compare the determinants of similarity-based access to 
memory and the determinants of the subjective soundness and similarity match. Based on structuremapping theory, they predicted that subjective soundness would depend on the degree of shared relational structure, particularly high-order structure such as causal bindings. In contrast, they also predicted that memory retrieval would be highly sensitive to surface similarities such as common object attributes. To assess retrievability, they expose subjects to read a large set of stories and were later given a set of probe stories that resembled the original stories in systematically different ways, for example, purely relational analogies, surface-similarity matches or literal similarity matches.

Research since the mid 80's confirms the venerable tradition that similarity increases the probability of transfer (Anderson, Farrell \& Sauer, 1984; Holyoak \& Koh, 1987; Novick 1988; Pirroli, 1985; Reed 1987; Ross, 1987; 1989; Simon \& Hayes, 1976). At the same time research findings indicate that the roles of similarity in transfer are complex:

I) First, that accuracy of transfer depends critically on the degree of structural match, that match causal structures (Schumacher \& Gentner, 1988a, b; Holyoake \& Koh, I987)

2) Second, people do not often succeed in accessing structurally appropriate materials, even when they are in the long term memory. (Gick \& Hoyoak,1980, 1983);

3) Third, similarity-based reminding is often based on superficial commonalties and /or instead of structural commonalities (Gentner, Ratterman \& Forbus, 1993: 526).

Such findings suggest that there is more than one type of analogy and therefore there is the possibility of other types of analogies that seem to influence cognitive processes in transferring knowledge from a base towards a target, as for example, in comprehending a text. Genter et al (I993) distinguish between three types of analogy: literal similarity, analogy and surface similarity (mere appearances). In literal similarity both relational predicates and object attributes are shared. In analogy (also called structural similarity) high order, structural relations are mapped and inferences are drawn. In surface similarity or (mere appearance matches), only object-attributes and low order relation are shared. There is a general consensus that in similarity-based transfer there are certain core cognitive processes that are involved: I) accessing a potential analogy, 2) matching the base analogue with the target, 3) mapping further inferences from the base to the target, 5) evaluating the soundness of the analogy and 6) extracting the common structure for later use (J. Clement, I986; Gentner, I988a. It is on account of this that similarity based transfer is considered to involve four to six sub-processes: accessing, matching, evaluating, drawing inferences (with some adaptation of the analogy) and abstracting from the analogy (Genter, Ratterman \& Forbus, 1993: 527).

This brings us to the core claims of this paper: the suggestion that different kinds of similarity use different cognitive sub-processes. In so doing, they exert different effects on accessing and abstracting or drawing of inferences. Specifically this paper makes the submission that individuals are more likely to access and remember surface features in a text comprehension more than they would in respect of texts that may be structurally similar but have different surface features and context. Similarly even though accessing structurally similar text may be initially difficult, yet when they succeed in doing so it is more consolidated.

\section{Results and Discussion}

\section{Research Question I: How is accuracy of recall of text dependent on the degree of surface similarities?}

In this question, even though our interest was first and foremost to assess the degree of surface similarity in retrieving information from text, we also evaluated the link between inference-drawing and surface similarity. 
On Inference-drawing: As predicted, students were able to make more inferences with respect to those stories that shared higher-order relational structures (the analogy matches) than those pairs of stories that did not (the FOR matches and surface matches). Comparing the three variables in terms of mean scores, Analogy matches received 4 point mean rating; surface similarity 2.8 and first order rule 2.6 rating. These findings were interpreted to mean that the three types of similarity matches have differential effects on inference. Analogy especially across-domain analogy is critical when it comes to higher level abstraction when one has to map higher orders from a base knowledge to target knowledge. This finding contradicts that of Holyoak (1983, 1987) that spontaneous transfer in problem solving without some form of instructional manipulation is very rare. Indeed in an unpublished doctoral dissertation of this author in 1998, the findings corresponded and corroborated with that of Holyoak (ibid). Out of a total of 240 Senior High School students that took part in the experiment to solve the radiation problem and other isomorphic problems only about 30\% were able to solve the problem fully. However, when later exposed to didactical intervention on how to use analogy in problem solving, many of the students $(57 \%)$ were able to solve similar problems after the instructional event.

This notwithstanding, this same finding which contradicts that of Holyoak (ibid) also corroborates other study, such as Day and Gentner (2007) study of non-intentional text comprehension. How do we explain this apparent inconsistency? The inconsistency seems to suggest that analogical mapping though often viewed as an explicit deliberative process can sometimes operate without intent or even awareness: and this is especially so in text comprehension. For example, when given a text such as: 'the robbers crossed the river and robbed the bank'. Most readers can readily make the relevant mapping in this context, that the bank being referred to here is not the bank of a river, (even though river is mentioned) but where money is saved because of the context of the subject 'robbers'. Thus the inference made of the bank (in which money is kept and not that of a river) in this instance is made on the basis of sound inference by an analogy that is not based on surface similarity per se. Thus if this finding in this first experiment is anything to go by, it does seem to suggest some plausible answer to our first research question: How is accuracy of recall of text dependent on the degree of surface similarity? The answer that this finding seems to suggest is this: even though analogy matches were not well recalled compared to surface matches, they were nonetheless rated as inferential.

\section{Reminding}

The scores on this dimension (measured on a score of 2 or better) show a different pattern: on all three measures, Analogy (AN):0.5; surface similarity (SS) 0.8 and first order reminding (FOR) 0.3. On all three measures on reminding, the AN matches were less effective than the SS. Thus, whereas surface similarity was more effective in recall, analogy and first analogy order matches received fewer score. What the result seems suggest is that surface similarity is quite crucial in recall as opposed to making sound inferences. They produced greater proportion of remindings followed by AN and then by FOR matches supporting surface similarly superiority in retrieval. This shows a remarkable difference in the case of inference. In the case of inference-drawing as predicted by structure-mapping theory, common higher-order relational structure is a crucial determinant of the subjective goodness of an analogy. Subjects rated AN matches as more sound than FOR matches whilst rating SS matches as no better than FOR matches. Thus, the result suggest that inference-drawing is not determined by the number of common features but seem to suggest that common higher-order relational structure is more crucial. Even though analogy matches were not well recalled, they were rated as inferential. The opposite was true for surface matches. This corroborates with the findings of Gentner et al (1993), Day and Gentner (2007), Reed (1987), Novick (1988). 


\section{Research Question 2: Do people fail to infer structurally appropriate analogies in text comprehension?}

The purpose of this second research question among others was to ensure that the subjective inference that were made by subjects in research question one (I) was not the result of extraneous factors such as cueing (that is students being biased by the experimenter to look out for higher order inferences ) as they read the texts in the first experiment. One of the main criticisms against the 'evidence' for transfer is that subjects are subtly manipulated either through direct instruction or other form of cuing. Hence, this second question was to cross check gauging and biasing students. It was to test why subjects often fail to access structurally appropriate analogies as compared to surface similarity analogies without any direct hint. They were given the materials in the first experiment and were asked to make inference. Students were measured along the following dimensions:

Inference: They were given the instruction: 'humans have intuitions about resemblances that seem weak or irrelevant. In this second question of the experiment, use your intuitions about when two situations match well enough to make a strong argument...'

Sound match: one in which essential aspects of the stories match: that is strong enough that one can infer or predict things about the second story from the first.

Similarity: subjects read stories and rated them on I-5 point rating scale with $5=$ extremely similar and $\mathrm{I}=$ extremely dissimilar.

Reminding: the procedure was as in Research Question I.

Inference: As predicted, in terms of mean rating, subjects scored a mean point of 5.4 on literal similarity that had common attributes and 5 point score on analogy with no common attributes on the YES, while there were low score for surface similarity of 2.3 on common attributes and 2.0 on first order relations with no common attributes on the NO for $67 \%$ the participating students. On literal similarity, there was a score of $5.2, \mathrm{AN}=4.8, \quad \mathrm{SS}=3.4$ and $\mathrm{FOR}=2.8$ students.

Similarity: Here the two groups of students ratings appeared to have shown some level of sensitivity to two types of commonalities: relational and object-attribute (see figure below). Literal similarity matches had score of $(M=4.60)$ over $A N$ matches of $(M=4.10)$, of AN matches of over SS $(\mathrm{M}=3.45)$, and of SS matches over FOR matches $(\mathrm{M}=2.93)$. The comparison between similarity and inference (soundness) is quite revealing: similarity like inference is sensitive to the presence of higher order relational structure.

\section{a) Mean soundness (inference rating) for four similarity types in Research Question 2}

\section{INFERENCE}

$\mathrm{LS}=(\mathrm{M}=5.4)$

$\mathrm{AN}=(\mathrm{M}=5)$

$\mathrm{SS}=(\mathrm{M}=2.3)$

$\mathrm{FOR}=(\mathrm{M}=2.0)$

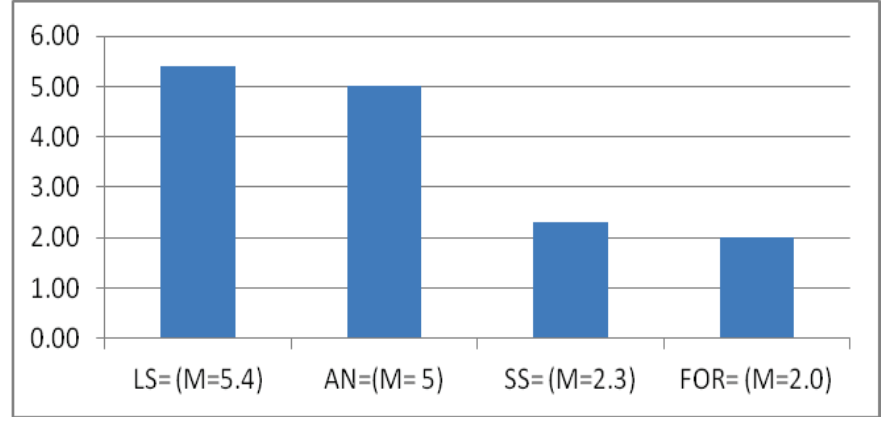

b) Mean similarity ratings for four similarity types in Research Question 2 
$\mathrm{LS}=(\mathrm{M}=4.60)$

$\mathrm{AN}=(\mathrm{M}=4.10)$

$\mathrm{SS}=(\mathrm{M}=3.45)$

$\mathrm{FOR}=(\mathrm{M}=2.93)$

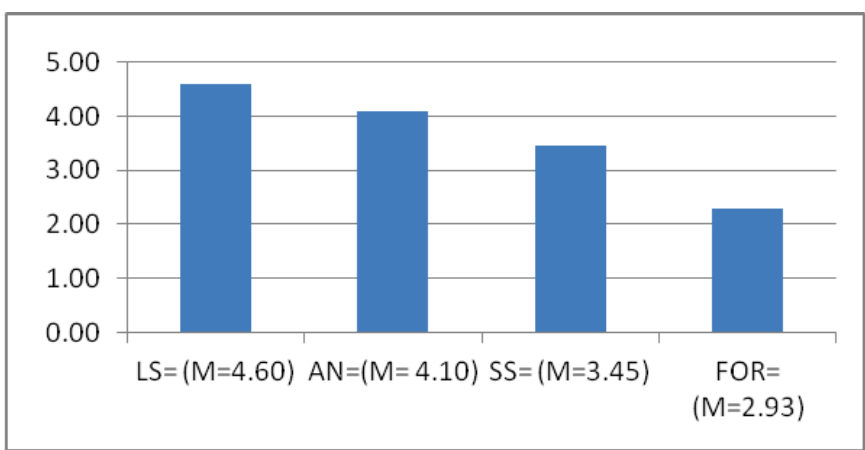

c) Mean Reminding ratings for four similarity types in ResearchQuestion 2

$\mathrm{LS}=(\mathrm{M}=.52)$

$\mathrm{AN}=(\mathrm{M}=.8)$

$\mathrm{SS}=(\mathrm{M}=.49)$

$\mathrm{FOR}=(\mathrm{M}=.05)$

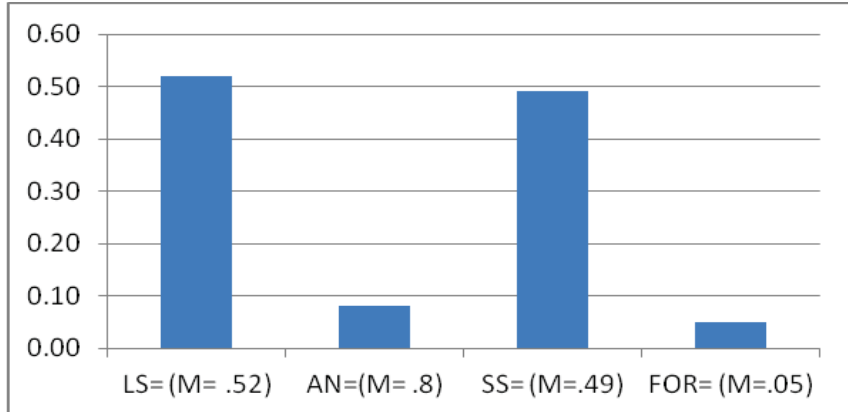

Reminding: this pattern was different from that of inference and similarity. Object commonalities strongly contributed to memory access and higher order relational had little effect. The scores were as follows: LS $(\mathrm{M}=.52)$ and $\mathrm{SS}(\mathrm{M}=.49)$ matches than $\mathrm{AN}(\mathrm{M}=.8)$ and FOR $(\mathrm{M}=.05)$.

Thus the answer to the second research question of this study: Do people fail to infer structurally appropriate analogies in text comprehension? The findings here seem to suggest that in text comprehension, people infer structurally appropriate analogies through the differential effects of different types of similarities, such as literal similarity matches which often are greater than analogy matches, just as the latter is greater than surface similarity and surface similarity takes precedence over first order relations. All these types of similarities may be sensitive to both relational and object-attribute commonalities. The plausible answer to this second research question seems to be this: No, in text comprehension, people may infer structural analogy through a hierarchy of cognitive sub-processes of the different analogy types. In terms of reminding, higher order relations had negligible effect as compared to object commonalities which had a strong influence on memory access.

Research Question 3: Why is transfer likely to occur to the extent in which base and target share surface similarity?

Inference (soundness): Consistent with prediction, the rate of inference reflected the degree of relational overlap. The AN matches $(M=3.80)$ were rated as significantly more sound than the SS matches $(M=$ 2.28). The soundness advantage of analogy over superficial similarity was greater for objects-only matches than for SS matches. 
Similarity: $\mathrm{AN}$ matches $(\mathrm{M}=4.04)$ were rated as more similar than $\mathrm{SS}$ matches $(\mathrm{M}=3.24)$ and OO matches $(\mathrm{M}=2.33)$ (within-group $\mathrm{t}(\mathrm{IO})=6.53$ and $\mathrm{t}(\mathrm{IO})=2.99$ respectively. SS matches were rated more similar than $\mathrm{OO}$ matches $\mathrm{t}(\mathrm{I} 8)=3.52$. Also for soundness, the difference between $\mathrm{AN}$ and $\mathrm{OO}$ matches was greater than the difference between AN and SS matches $\mathrm{t}(20)=2.8 \mathrm{I}$

Reminding: Again in this experiment as in the previous one, the pattern of the reminding differs from that of inference (soundness) and similarity. Both SS matches $(M=.50)$ and $\mathrm{OO}$ matches $(\mathrm{M}=. \mathrm{I} 4)$ were better retrieved than $\mathrm{AN}$ matches $(\mathrm{M}=.05)$ within-subject $\mathrm{t}(20)=6.60$ and $\mathrm{t}$ $(20)=2.76$. SS matches were better retrieved than $\mathrm{OO}$ matches $\mathrm{t}(50)=4.58$. The anchoring LS were highly retrievable as expected $(M=.60)$.

The pattern of the quality of recall was similar. The mean quality of recall was significantly higher for $\mathrm{SS}$ matches $(\mathrm{M}=\mathrm{I} .42)$ than for $\mathrm{OO}$ matches $(\mathrm{M}=.44), \mathrm{t}(20)$. Quality of recall was higher for both SS matches and $\mathrm{OO}$ matches than for AN matches (M=.I9), $\mathrm{t}(20)$. As expected, LS matches received higher rating $(\mathrm{M}=\mathrm{I} .76)$. The difference between the $\mathrm{AN}$ and the SS matches was greater than the difference between the AN and $\mathrm{OO}$ matches $(\mathrm{t}(20)=4.6 \mathrm{I})$.

\section{Constraints on these Effects}

The results of the findings indicate that analogical processes are capable of influencing text comprehension without cueing subjects. However, it must be pointed out that these findings are based on a relatively small set of experimental materials that were designed to test for such effects. Much research work remains to be done to isolate the scope of these kinds of processes. In fact, the present findings are quite surprising (as mentioned earlier on), when one takes cognizance of the fact that quite a number of studies have shown little or no spontaneous transfer from single instances (Gick\& Holyoak, 1980, 1983; Holyoak \& Koh, 1987; Keane,1988; Ross, 1987, 1989). Additionally, many studies have shown that comparison of two or more instances can produce impressive transfer when isolated instances cannot (Catrambone \& Holyoak, 1989; Gentner, Loewenstein, \& Thompson, 2003; Gick \& Holyoak, 1983). This appears to indicate that this kind of effect may often require abstracted schemas rather than concrete instance representations. We can look to differences between the experiments presented in this study and previous research for clues about possible constraints on our effects. One potentially critical factor is the degree and kind of similarity between the base and target passages. Previous research such as (Gentner\& Landers, 1985; Gentner et al., 1993; Holyoak \& Koh, 1987), have established the importance of surface similarity in explicit analogical reminding giving indication of a dissociation between the kind of similarity that supports alignment and inferencing (i.e., structural similarity) and the kind that supports memory access to prior instances (i.e., surface similarity-similar characters, objects, and settings). In the present study, the story pairs were high in both structural and surface similarity.

Thus, it could be the case that spontaneous inferencing requires a high degree of surface similarity. However, there is some evidence (Catrambone, 2002; Gentner et al., I993; Wharton, Holyoak, \& Lange, 1996) that purely structural similarity also contributes to analogical reminding. This raises the question that possibly spontaneous transfer might occur even without surface similarity. These findings are therefore not absolute. One significant way in which the present study differs from past research is in the kind of inferences involved (Day \& Genter, 2007).

\section{I0. Conclusion}

This study began with the fundamental claim that analogy is central to human cognition. Our hypothesis was that there are different types of analogies that influence the processes of transfer, 
inference drawing and memory access. This implies that the account of the effect of analogies requires making fine distinctions in analogies in transfer and text comprehension. This study specifically found that there is dissociation between the matches that people get from memory and those matches they want. Memory accessibility was found to be strongly influenced by surface commonalities but weakly influenced by structural commonalties. The contrast was that inference-drawing was strongly influenced by structural commonalties and not in any way influenced by surface commonalities. The implication of this finding is that in text comprehension, readers are more likely to access surface commonalities most of the time, yet in making inference and text elaboration, it is more likely that readers would attend to higher order relations and not surface similarities non -intentionally without realising that they are doing so.

Inference-making: The findings of this study suggest the structure-mapping theory prediction which posits that subjective soundness of a similarity match is determined by the degree of relational structure overlap. The three experiments of the study bear this out. First when common relations are added as in the first experiment, it increases the perceived soundness of a match. In every case where a precise comparison is possible the probability of inference-making is increased with the relational commonalities. For example, in both cases of research Question I and 2, when higher relations were added, analogies were judged more sound than first order matches. Similarly in research question 2, literal similarity matches were judged more sound than surface matches. In research question 3, surface matches were judged more sound than objects only matches.

The second finding is this: adding higher-order commonalities to a first-order relational match increases inference-drawing (soundness) more than adding object commonalties. The three experiments bear out that the inference of analogical matches is substantially higher than that of surface matches.

The third evidence that this study seems to underscores is also this: the addition of objectattribute commonalities fails to increase (inference-making) soundness. There is compatibility of these findings with other study such as Gentner et al (1993), Gentner and Clement (1988).

Resonance Models of Activation: Our results seem to be compatible with those studies that have examined the role of general memory processes in the generation of inferences and maintenance of coherence in text, largely focusing on "resonance" models of memory activation (e.g., Gillund \& Shiffrin, 1984; Hintzman, 1986; Ratcliff, 1978; Day \& Gentner, 2007). 'Resonance' models make the claim that there is selective parallel activation from the long term memory. This selective activation is contingent upon common overlap with concurrent working memory. According to Day and Gentner (2007), these approaches have been successfully used in the explanation of anaphor resolution and reactivation of distant, but relevant, information from earlier in a text (cf. O'Brien, 1995). Based on such data, it does not seem unreasonable to hypothesize that these kinds of processes could play a role in the effects found in the present experiments. The high degree of similarity between the source and target passages could have allowed resonance-type activation of the base passage as a whole, or even have supported more localized element-to-element mappings in the course of reading (Day \& Gentner, 2007: 46).

Similarity-based Access: The results for access were the opposite of the results for inferencemaking. Subjects had the tendency not to retrieve the matches they considered most sound. Rather they were more likely to access surface matches. There are three lines of evidence in this respect: a) combining object attributes to a match increased the proportion of retrieved. In the first two experiments, recall of surface matches was greater than recall for FOR matches. In the second experiment, recall of literal similarity matches was greater than recall of analogical matches; b) adding common object attributes contributes more to retrievalilibty than adding common higher-order reactions. In all three experiments recall of surface similarity was far greater than recall of analogical matches. The proportions of surface matches retrieved across three experiments were substantially higher than retrieval rate for analogies. 
Similarity: Accessibility and subjective soundness (inference-making) are both aspects of similarity. Adding subjective similarity resembled soundness in respects of its sensitivity to common structural relations. Adding relational information increased subjective similarity. Literal similarity matches were considered more similar than surface matches (Research Question 2); analogical matches were considered more similar than FOR-matches (Research Question t 2) and surface matches were considered object-only matches (Research Question 3). Another line of evidence is the comparative addition argument. Comparing the effect of higher-order commonalties verses adding object commonalties we find the following: analogies are rated as more similar than surface matches in both experiment 2 and 3. However, unlike soundness, similarity is increased by adding object commonalities and for this literal similarity matches are considered more similar than analogy matches; whilst surface matches are also considered more similar than For matches (Research Question) So it looks like both surface similarity contribute to subjective similarity.

Thus as per the findings of this study, one can conclude with some level of plausibility that there are different types of analogies (similarity) that have differential effects on both access and inferential soundness. Whereas across-domain or far transfer analogy affects inference and elaboration, literal similarity or within-domain analogy has influence on retrievability.

\section{References}

Anderson, J.R., Farrell, R. \& Sauers, R.(1984). Learning to programme in LISP. Cognitive Science, 8, 87-I29

Catrambone, R. (2002). The effects of surface and structural feature matches on the access of story analogs. Journal of Experimental Psychology: Learning, Memory, \& Cognition, 28, 318-334.

Catrambone, R., \& Holyoak, K. J. (1989). Overcoming contextual limitations on problem-solving transfer. Journal of Experimental Psychology:Learning, Memory, \& Cognition, I5, I I47-I 156.

Clement, J.(1986). Methods of evaluating the validity of hypothesised analogies. Proceedings of the Eighth Annual conference of of the Cognitive Science Society (pp.223-234), Amherst, MA. Hillsdale, NJ: Erlbaum Clement, C. A., \& Gentner, D. (I99I). Systematicity as a selection constraint in analogical mapping. Cognitive Science, I5, 89-I32.

Day, S.B. \& Gentner, D (2007).Nonintentional analogical inference in text comprehension. Memory \& Cognition 35 (I) $39-49$

Detterman, D \& Sternberg, R.J (I993). (Eds.), Transfer on trail: intelligence, cognition and instruction. NJ: Ablex

Forbus, K. D., Gentner, D., \& Law, K. (1995). MAC/FAC: A model of similarity-based retrieval. Cognitive Science, 19, I4I-205.

Gentner, D.(1983). Structure-mapping: A theoretical framework for analogy. Cognitive Science, 7, I55-170

Gentner, D. (2003). Analogical reasoning, psychology of. In L. Nadel (Ed.), Encyclopedia of cognitive science (Vol. I, pp. I06-II2). London:Nature Publishing.

Gentner, D., \& Clement, C. (1988). Evidence for relational selectivity in the interpretation of analogy and metaphor. In G. H. Bower (Ed.), The psychology of learning and motivation: advances in research and theory (Vol.22, pp.307-358). New York: Academic Press

Gentner, D., Holyoak, K. J., \& Kokinov, B. N. (Eds.) (200I). The analogical mind: Perspectives from cognitive science. Cambridge, MA: MIT Press.

Gentner, D., \& Landers, R. (1985). Analogical reminding: A good match is hard to find. In Proceedings of the International Conference on Cybernetics and Society (pp. 607-6I3). New York: IEEE Press

Gentner, D., Loewenstein, J., \& Thompson, L. (2003). Learning and transfer: A general role for analogical encoding. Journal of Educational Psychology, 95, 393-405.

Gentner, D., \& Markman, A. B. (1997). Structure mapping in analogy and similarity. American Psychologist, 52, 45-56.

Gentner, D., Ratterman, M.J.,\& Forbus, K.D.(1993). The roles of similarity in transfer: Separating retrievability from inferential soundness. Cognitive Psychology, 25, 254-575 
Gick, M.L., \& and Holyoak, K.J. (I983). Schema induction and analogical transfer. Cognitive Psychology, I5, I38.

Gick, M.L \& Holyoak,K.J. ( I 987). The cognitive basis of knowledge transfer. In S.M. Cormier \& J. D Hagman (Eds.). Transfer of learning, contemporary research and applications (pp.9-42) New York: Academic Press.

Gillund, G., \& Shiffrin, R. M. (I984). A retrieval model for both recognition and recall. Psychological Review, 9I, I-67.

Goswami, U. (1992). Analogical reasoning in children. Hillsdale, NJ: Erlbaum

Halford, G.S. (1992). Analogical reasoning and conceptual complexity in cognitive development. Human Development, 35, 193-217.

Hintzman, D (1986). 'Schema abstraction ' in a multiple-trace memory moel. Psychological Review, 93, 528-55 I

Hintzman, D (1988). Judgemnets of frequency and recogntiton memeory in a multipe-trace memory model. Psyhcological Review, 95, 528-55I

Holyoak, K.J, (I985).The pragmatics of analogical transfer. In G.H. Bower (Ed.), The psychology of learning and motivation: Advances in research and theory (Vol. 19,pp 59-87). New York: Academy Press.

Holyoak, K. J \& Koh., K. (1987). Surface and structural similarity in analogical transfer. Memory \& Cognition, I5 332-340.

Holyoak, K. J., \& Thagard, P. (I995). Mental leaps: Analogy in creative thought. Cambridge, MA: MIT Press.

Holyoak, K. J., \& Thagard, P. (I989). Analogical mapping by constraint satisfaction. Cognitive Science, I3, 295 355.

Hummel, J. E., \& Holyoak, K. J. (1997). Distributed representations of structure: A theory of analogical access and mapping. Psychological Review, I04, 427-466.

Keane, M. T. (1988). Analogical problem solving. New York: Wiley.

Kokinov, B., \& French, R. M. (2003). Computational models of analogy making. In L. Nadel (Ed.), Encyclopedia of cognitive science (Vol. I, pp. I I3-I 18). London: Nature Publishing.

Markman, A. B. (1997). Constraints on analogical inference. Cognitive Science, 2I, 373-4I8.

Novick, L. R. (1988). Analogical transfer, problem similarity, and expertise. Journal of Experimental Psychology: Learning, Memory, and Cognition, I4, 510-520.

O’Brien, E. J. (1995). Automatic components of discourse comprehension. In R. F. Lorch, Jr. \& E. J. O’Brien (Eds.), Sources of coherence in reading (pp. I59-176). Hillsdale, NJ: Erlbaum.

Pirroli, P.(I985). Problem solving analogy and skill acquisition in the domain of programming. Unpublished manuscript.

Ratcliff, R. (1978). A theory of memory retrieval. Psychological Review, 85, 59-I08.

Reed, S.K (1987). A structure-mapping model for word problems. Journal of Experimental Psychology: Learning, Memory and Cognition, I3, 629-639

Reeves, L. M. \& Weisberg, R.W (1994). The role of content and abstract inofrmation in analogical transfer. Psychologcial Buletin II5 (3), 38I-400

Ross, B.H.( 1987). This is like that: the use of earlier problems and the separation of similarity effects. Journal of Experimental Psychology: Learning, Memory and Cognition, I3, I24-I39

Ross, B.H. (1989). Remindings in learning and instruction. In S. Vosniadou \& A. Ortony (Eds.)., Similarity and analogical reasoning (pp.438-469). New York: Cambridge University Press.

Schumacher, R.M \& Gentner, D.(I988a). Remembering causal systems: Effects of systematicity and surface similarity in delayed transfer. Proceedings of Human Factors Society $32^{\text {nd }}$ Annual Meeting (pp I27I-I275). Anaheim, CA, Santa Monica, CA: Human Factors Society

Schumacher, R.M. \& Gentner, D.(I988b). Transfer of training as analogical mapping. IFEE transactions on systems. Man, and Cybernetics, I8, 592-600

Simon, H. A \& Hayes, J.R 1976). The understanding process: Problem isomorphs. Cognitive Psychology, 8, I65I90

Wharton, C. M., Holyoak, K. J., \& Lange, T. E. (1996). Remote analogical reminding. Memory \& Cognition, 24, 629-643. 